\title{
Uncovered
}

\section{Hierarchical electrospun PIM nanofibers decorated with ZnO nanorods for effective pollutant adsorption and photocatalytic degradation}

\section{Kugalur Shanmugam Ranjith*, Bekir Satilmis, Tamer Uyar}

Institute of Materials Science \& Nanotechnology and UNAM-National Nanotechnology Research Center, Bilkent University, Ankara 06800, Turkey

ranjuphy@buc.edu.in (K.S. Ranjith), tamer@unam.bilkent.edu.tr (T. Uyar)

Hierarchical functionality with the nanostructural features is interesting since it is plausible to have unique physicochemical functionalities with applications in various fields such as detectors, catalyst, energy harvesters, etc. Designing feasible architectures under the requirement of different nanostructural features as free-standing webs was exhibited with multifunction properties along with its advantages in the combination of fiber morphology and structural feature. Polymers of intrinsic microporosity (PIMs) have sparked considerable interest in selective adsorption and separation applications due to the structural diversity along with their highly porous nature [1]. PIM-1, is the first member of this class, has specific interactions with the organic functionalities, which make it a suitable platform for the selective adsorption, separation and catalytic applications in industrial process. It has exhibited outstanding performance in gas separation applications in the membrane form [2]. PIM-1 has shown high affinity for neutral species and the affinity can be tailored by chemical modification [3]. Modifying the PIM-1 to a nanofibrous web implies effective applicability in adsorption, detection and energy storage applications. The electrospun PIM-1 nanofibers exhibited effective adsorption of aniline from

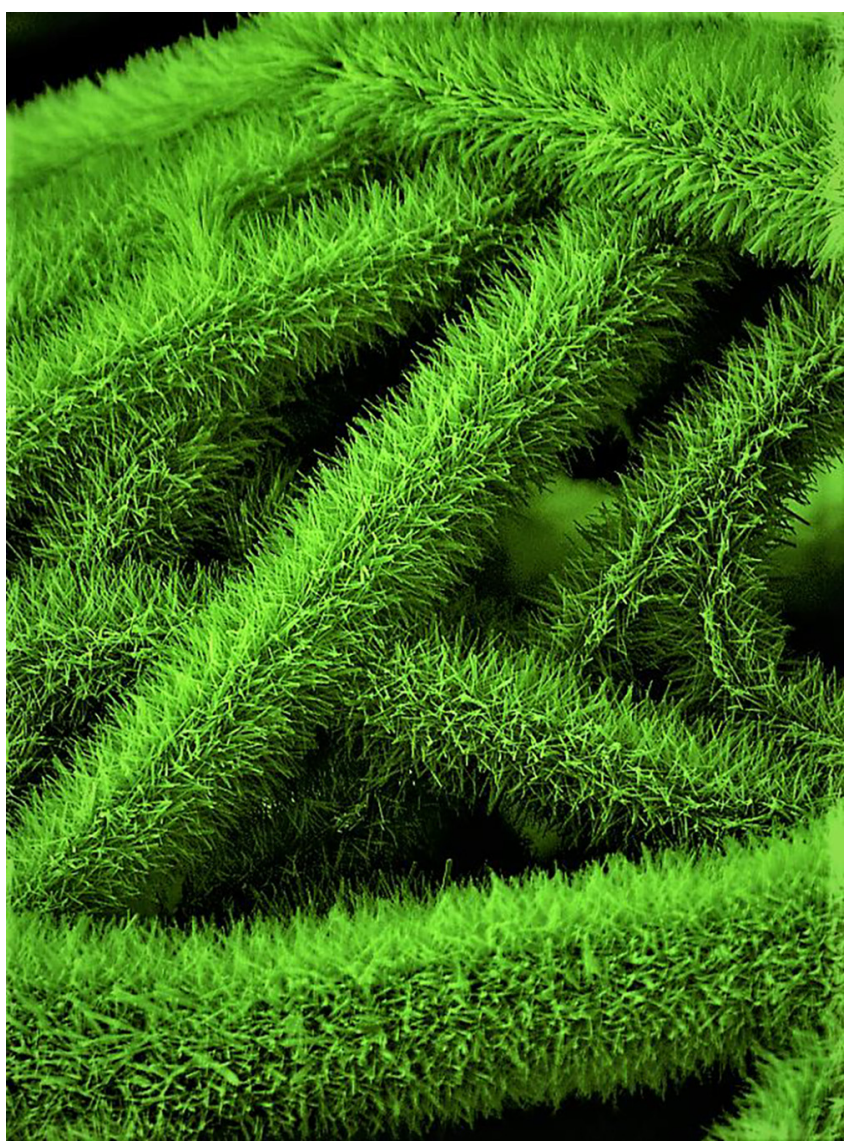

the aqueous medium and from the air [4], and some neutral dyes [5]. Moreover, functionalization of the PIM-1 nanofibers is possible for improving the adsorption affinity in the aqueous medium. For instance, by chemically modifying the nitrile group of PIM-1, hydrolyzed PIM-1 electrospun nanofibers can provide enhanced adsorption and separation performance [6].

In our group, one of the main focuses is electrospinning of polymeric and inorganic nanofibers/nanowebs as a membrane for the effective and selective adsorption, separation and catalytic processes. Electrospinning is a versatile technique which 
uses the high electric field to produce nanofibrous web in the form of the membrane with the 1D nanostructural features with the fashionable surface functionality. The production of the hydrolyzed PIMs in the form of the nanofibrous web is attractive by its selective surface functional groups, high surface area, porous nature and typical architecture [6]. The PIM-based electrospun polymeric nanofibers were effectively utilized as a platform for adsorbing the pollutants and used as filters to separate the organic pollutants. Chemical modification of PIMs is essential to attain a fine control over the selectivity as well as the adsorption capacity. Transforming the PIMs into hydrolyzed PIMs (HPIMs) will modify its adsorption ability against the cationic species [6]. In addition, amine modification will tune the ability against anionic species [7]. Modifying the PIMs has been interesting nowadays over the selectivity toward the anionic and cationic functionalities to separate them. The functional role of $\mathrm{ZnO}$ was to promote the effective photocatalytic nature with its promising defect functionalities which lead to visible responsiveness [8]. Coupling the $\mathrm{ZnO}$ nanorod (NR) nanostructures with the electrospun HPIM nanofibers initiated the effective adsorption of cationic dye molecules and under the photo irradiation the adsorbed dyes reacted with the photogenerated reactive oxygen species produced from $\mathrm{ZnO}$ surface, degrading the dye molecules. Adopting the porous features and functionality of surface nanofibers effectively adsorb the pollutants to its surface and surface decorated ZnO NRs led to degradation of the adsorbed pollutants under photoirradiation. By modifying the PIM surface features with the different inorganic integrities as a hybrid heterostructural hierarchical finish may lead to the advanced applications in future. Researchers have to pay attention on constructing the hybrid inorganic-organic heterostructural membranes with the tunable structural and functional features to promote the commercial utility.

This cover image of the issue refers to the scanning electron microscopy image of HPIM nanofibers decorated with the $\mathrm{ZnO}$ NRs from the combination of electrospinning and surface functionalization process. The hierarchical decoration ZnO NRs on the HPIM nanofibers with the atomic level interaction of $\mathrm{ZnO}$ seed layer through atomic layer deposition (ALD) process offered a uniform nucleation site on the HPIM surface for the ZnO NR growth. The hierarchical structure over the HPIM electrospun nanofibrous membrane was attempted to couple the high pollu- tant adsorption nature of HPIMs and high photodegradation of $\mathrm{ZnO}$ in a single platform as a hierarchical web for the effective remediation-based application. Various attempts were initiated to grow the NRs on the HPIM surface, but the hydrophobic nature of the fiber surface leads to the poor interaction and non-uniform seed layer functionality which leads to the poor NR growth. But while we prefer the ALD process, it initiates the thin uniform level of seed nucleation distribution which offers the preferred growth of the ZnO NRs to be wrapped around the polymeric fibers. The unique arrangement innovates in this approach on the effective and selective adsorption of pollutants from the wastewater and later it degrades under photo irradiation. Using this protocol, different set of hybrid functionality of inorganic features can be built over the HPIM surface for feasible multifunction applications. The hierarchical features of the nanofibrous web-based hybrid system lead to the development of the wastewater remediation activities. Constructing the hybrid hierarchical architecture can serve an effective and efficient role in the multifunctional application in the field of catalyst, detectors, and electronics with compatibility and long-term sustainability.

This year's cover competition is brought to you
in association with ZEISS. As the world's only
manufacturer of light, X-ray, electron and ion
microscopes, ZEISS offers tailor-made micro-
scope systems for materials research, academia
and industry.
Visit www.zeiss.com/microscopy to learn more.
Visit http://www.materialstoday.com/materials-
today-cover-competition-2017/ to see the all the
winning images.

\section{Further reading}

[1] N.B. McKeown, P.M. Budd, Chem. Soc. Rev. 35 (2006) 675-683.

[2] L.M. Robeson, J. Membr. Sci. 320 (2008) 390-400.

[3] B. Satilmis, P.M. Budd, J. Colloid Interface Sci. 492 (2017) 81-91.

[4] B. Satilmis, T. Uyar, J. Colloid Interface Sci. 516 (2018) 317-324.

[5] C. Zhang, P. Li, B. Cao, J. Appl. Polym. Sci. 133 (2016) 43475 (1-10).

[6] B. Satilmis, P.M. Budd, T. Uyar, React. Funct. Polym. 121 (2017) 67-75.

[7] B. Satilmis, T. Uyar, Appl. Surf. Sci. 453 (2018) 220-229.

[8] K.S. Ranjith et al., Catal. Sci. Technol. 7 (2017) 1167-1180. 